\title{
Impact of removable partial denture prosthesis on chewing efficiency
}

\author{
Marion BESSADET, Emmanuel NICOLAS, Marine SOCHAT, Martine HENNEQUIN, Jean-Luc VEYRUNE
}

Clermont Université, Université d'Auvergne, Centre de Recherche en Odontologie Clinique; CHU Clermont-Ferrand, Service d'Odontologie, Hôtel-Dieu France, Clermont-Ferrand, France.

Corresponding address: Dr. Emmanuel Nicolas - UFR Odontologie, CROC - 11 bd Charles de Gaulle - 630000 Clermont - Ferrand, France - Phone: 0473 177382 - Fax: 0473177388 - e-mail: emmanuel.nicolas@udamail.fr

Submitted: January 16, 2013 - Modification: June 3, 2013 - Accepted: July 4, 2013

\section{ABSTRACT}

\begin{abstract}
$\mathrm{R}^{\mathrm{mat}}$ emovable partial denture prostheses are still being used for anatomic, medical and economic reasons. However, the impact on chewing parameters is poorly described. Objectives: The objective of this study was to estimate the impact of removable partial denture prosthesis on masticatory parameters. Material and Methods: Nineteen removable partial denture prosthesis (RPDP) wearers participated in the study. Among them, 10 subjects were Kennedy Class III partially edentulous and 9 with posterior edentulism (Class I). All presented a complete and full dentate opposing arch. The subjects chewed samples of carrots and peanuts with and without their prosthesis. The granulometry of the expectorated boluses from carrot and peanuts was characterized by median particle size (D50), determined at the natural point of swallowing. Number of chewing cycles (CC), chewing time $(C T)$ and chewing frequency $(C F=C C / C T)$ were video recorded. Results: With RPDP, the mean D50 values for carrot and peanuts were lower [Repeated Model Procedures (RMP), $F=15, p<0.001$ ] regardless of the type of Kennedy Class. For each food, mean CC, CT and $C F$ values recorded decreased ( $R M P, F=18, F=9$, and $F=20$ respectively, $p<0.01$ ). With or without RPD, the boluses' granulometry values were above the masticatory normative index (MNI) determined as 4,000 $\mu \mathrm{m}$. Conclusion: RPDP rehabilitation improves the ability to reduce the bolus particle size, but does not reestablish fully the masticatory function. Clinical relevance: This study encourages the clinical improvement of oral rehabilitation procedure.
\end{abstract}

Keywords: Rehabilitation. Removable partial denture. Mastication.

\section{INTRODUCTION}

According to some studies, dentists often overestimate the functional consequences of edentulous areas. In 1974, Levin ${ }^{8}$ described the "28 teeth syndrome", which led to systematically replacing any missing teeth. Subsequently, studies tended to emphasize the shortened arch concept, which corresponds to a denture comprising 3 to 5 functional units (a functional unit being established by a pair of premolars or molars in occlusion) and some authors still tend to defend this concept ${ }^{6,17}$. According to Käyser, 12 anterior teeth and 8 premolars would be necessary and sufficient to ensure the mandibular stability required for secure deglutition without aspiration, provided that these teeth are efficient ${ }^{6,17}$. However, increasing the masticatory surface is not the only argument for absent tooth replacement: esthetics and wedging of opposing and neighboring teeth are also important, and thus reasons that lead both patients and practitioners to replace missing teeth. It also raises the question about preserving the integrity of temporomandibular joints.

In the case of partial edentulism, and based on clinical examination, rehabilitation of the oral function can be obtained with fixed or removable prosthodontics. The removable partial denture prosthesis (RPDP) must be able to restore the chewing function, esthetics and phonetics. It compensates for partial edentulism ${ }^{6}$. Understanding of the biomechanics involved (appreciation of the tissue's double nature - teeth and oral tissue - and thus of different behaviors) allows the practitioner 
to design a removable partial denture prosthesis satisfying the requirement of balance during the function, or in other words establishing and maintaining lift, stabilization and retention (the Housset triad). With these imperatives taken into account, and depending on the number of teeth lost and the type of edentulous areas (bounded by remaining teeth or without posterior tooth support), the constraints on the prosthesis will be different and functional rehabilitation altered. Effectively, during mastication, natural or artificial teeth are not simple tools that mechanically reduce the food to particles and mix saliva and the food to produce a bolus, easy to swallow. They also are essential to the neuromotor control of chewing and swallowing, through the sensory receptors ${ }^{10}$. Any oral disease that affects the numbers, the structure or the position of the teeth is supposed to have an impact on chewing and, in turn, on nutrition. A physiological approach is thus necessary to measure to what limits the edentulous status and their oral rehabilitation could affect the chewing function. Calculating food bolus granulometry collected before swallowing, associated with analysis of the kinematic parameters developed to produce this bolus, could help to distinguish patients with normal mastication from those with seriously impaired mastication ${ }^{1,18}$. Impaired chewing function leads to an increase of food bolus particle size, measured by the median particle size of the food bolus at swallowing. It has been shown that adults with impaired mastication could be distinguished from those with normal function if the median particle size of the bolus that they produced when chewing raw carrot reached a cut-off value of 4 $\mathrm{mm}$, called the masticatory normative indicator $(\mathrm{MNI})^{19}$. The adaptation of chewing behavior to food hardness can also characterize healthy mastication. Adaptation to increasing food hardness results in an augmented number of chewing cycles and an increase in the chewing sequence duration, with no modification of the chewing frequency (number of cycles per second) in healthy subjects ${ }^{3,7,13}$. The mean chewing frequency is slowed down in subjects with chewing deficiencies while eating any type of resistant food. Previous studies on the chewing ability of dentally impaired subjects showed that a decrease in the number of functionally paired teeth and oral rehabilitation with removable dentures were linked to a decrease in CT and CC values and to an increased D50 value ${ }^{9,14}$. Nevertheless, the physiological impact of RPDM rehabilitation has been seldom studied. Also, the objective of this work was to estimate the impact of partial edentulous areas rehabilitation by removable partial denture prosthesis with a metallic frame on chewing parameters ${ }^{4}$.

\section{MATERIAL AND METHODS}

\section{Study design}

The study was prospective and observational, and the subjects functioned as their own controls. Recruitment extended over 12 months in the Dental Department of the University Hospital of ClermontFerrand, France. The study included voluntary patients and was approved by the local ethics committee (CECIC, 2010/06; IRB number 5044). Each subject attended two times for mastication evaluation. The first evaluation was organized without any rehabilitation and the second with RPDP.

\section{Subjects}

The required sample size was estimated from a previous study that measured the carrot bolus granulometry in a group of edentulous subjects rehabilitated with implants procedure ${ }^{16}$. The mean D50 values of the carrot bolus decreased from $4,800 \pm 1,013 \mu \mathrm{m}$ (without rehabilitation) to $3,292 \pm 1,335 \mu \mathrm{m}$ (with implant rehabilitation). Calculations with the epiR package 0.9-30 were based on a difference of $1600 \mu \mathrm{m}$ and a common standard deviation value of $1,100 \mu \mathrm{m}$. That indicated the need for at least 9 subjects with Kennedy Class I and 9 patients with Kennedy Class III ( $a=5 \%$, $\beta=20 \%$ ). Thus, in 2010, 19 subjects from 24 to 79 years old; 6 men (mean age $=50.8 \pm 8.1$ years) and 13 women (mean age $=60.7 \pm 11.3$ years) participated in the study. Their prostheses had been in place for at least 2 months and they were able to eat without difficulty. Among them, 10 were wearing removable partial dentures prosthesis bounded by remaining teeth replacing at most 4 posterior teeth (maxillary or mandibulary, Kennedy Class III) and 9 were wearing posterior removable partial denture prostheses (Kennedy Class I). All subjects presented a complete, full dentate opposing arch. Two prosthodontics experts (calibrated to the evaluation) checked the conformity of the prosthetic design.

\section{Experimental procedure}

Two natural foods commonly used in mastication studies were used. Subjects were asked to chew with their prostheses three standardized samples of carrot (cylinders of $2 \mathrm{~cm}$ diameter adjusted in height to reach a weight of $4.0 \pm 0.5 \mathrm{~g}$ ) and non-salted draw peanuts (selected to reach a weight of $4.0 \pm 0.5 \mathrm{~g}$ ). Afterwards, the subjects were asked to repeat the operation without wearing their prostheses.

Video recording was used for evaluation of kinematic parameters ${ }^{12}$. A digital camera positioned in front of the subject (face-on) recorded a video of the face. All subjects were asked to close their eyes while the experimenter placed the food sample 
on the tongue so as to prevent recognition of the food sample. The subjects then had to close the mouth and teeth without contracting their muscles, keeping the food sample between the tongue and palate. When prompted by the experimenter, the subjects began chewing as naturally as possible. The subjects were then asked to chew three replicates of carrots and peanuts. The first replicate was completely masticated and swallowed for training. During this sequence, the chewing time was monitored by an investigator and was the baseline time for the following measurements. For the two other replicates, the patient was instructed to spit out each bolus when they thought it was ready to be swallowed. If there was a difference of more than $\pm 5 \mathrm{~s}$ between the chewing times of the swallowed and the two expectorated replicates, the patient was asked to chew a new piece of the test food.

\section{Bolus granulometry analysis}

Each chewed bolus (masticate) was collected in a container, rinsed with water in a $100-\mu \mathrm{m}$ sieve to eliminate saliva, and dried at $80^{\circ} \mathrm{C}$ for $30 \mathrm{~min}$. The bolus was then spread onto a transparent A4 sheet. The sheet was scanned to produce a 600-dpi image (Epson Perfection 4990 photo, Seiko Epson Corporation, Nagano, Japan). The images were then software-processed to evaluate food particle size and distribution (Powdershape ${ }^{\circledR}$ version 4.3.6 for Windows, 2005, Innovative Sintering Technologies, Vilters, Switzerland). For each masticate, the results were expressed in terms of the D50 value, characterizing the theoretical sieve size that would let through $50 \%$ of the particle weights ${ }^{14,19}$. Data were saved directly in an Excel ${ }^{\mathrm{TM}}$ file. Thus, D50 value decreased as food boluses contained more small particles. According to a previous study, the two D50 values recorded for each subject and each natural food were averaged, and D50 values for carrots above $4 \mathrm{~mm}$ were considered as coming from a subject with impaired mastication ${ }^{19}$.

\section{Kinematic parameters of mastication}

The recorded variables were chewing time (CT: the time in seconds between the moment the subject started to chew and swallowing, identified by the immediate swallow after the end of rhythm micro-rotary movements) and number of chewing cycles (CC: number of chewing actions during the CT period; this included all the rotary patterns, with and without lip closure). Chewing frequency (CF) was calculated as the ratio CC/CT. The evaluations of each kinematic parameter required an independent reading of each video recording by a calibrated observer who watched the recordings in random order $^{15}$. The internal reliability gave an ICC index ranging between $0.86-0.98(p<0.01)$ for chewing time and 0.92-0.97 for chewing cycles $(p<0.01)$. Calculation of external reliability gave an ICC index ranging between 0.97-0.98 for chewing time and from 0.90 to 0.97 for number of cycles. The method has previously been validated for healthy, fully dentate patients and for denture wearers ${ }^{5,12}$.

\section{Statistical analysis}

Statistical analysis was performed using IBM SPSS ${ }^{\circledR} 20$ software. Statistical significance was set at $p<0.05$. To evaluate the impact of RPDP rehabilitation on mastication parameters, the mean values of CC, CT, CF and D50 measured during mastication of carrot and peanuts were compared with and without RPDP by four repeated model procedures (RMP) (dependent factor: CC, CT, CF, D50; fixed factor: type of food; type of Kennedy Class). Values were expressed as mean \pm SD.

\section{RESULTS}

\section{Bolus granulometry}

With RPDP, the mean D50s were significantly decreased for carrot and peanuts (RMP, $F=15$, $p<0.001)$. These variations were independent of the type of Kennedy Class and type of food (Figure 1 ). With and without RPDP, the mean D50 values for carrot are above the MNI value of 4,000 $\mu \mathrm{m}$.

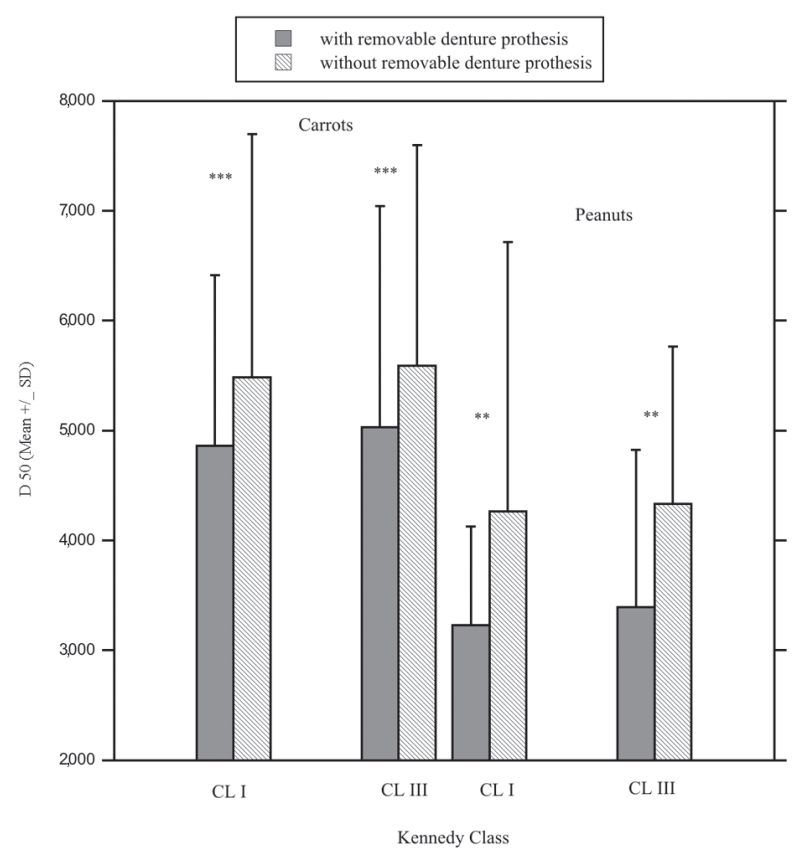

Figure 1- Mean variation \pm standard deviation of the granulometry of the carrot and peanut preswallowed bolus (D50, median size of bolus particle) whether a removable prosthesis was worn or not, and according to the type of edentulous areas. Repeated measure procedure was used for intergroup comparisons $\left({ }^{* * *} p<0.001,{ }^{* *} p<0.01\right)$ 
Table 1- Mean values \pm standard deviation of the kinematics parameters of the carrot and peanut mastication (CC, number of chewing cycles; $\mathrm{CT}$, chewing time; $\mathrm{CF}$, chewing frequency with $\mathrm{CF}=\mathrm{CC} / \mathrm{CT}$ ) with and without removable partial denture (RPD) rehabilitation and for each type of Kennedy class

\begin{tabular}{cccccc}
\hline & & \multicolumn{2}{c}{ Kennedy Class I } & \multicolumn{2}{c}{ Kennedy Class III } \\
& & Without RPD & With RPD & Without RPD & With RPD \\
\hline Carrot & CC & $44.2 \pm 26.9$ & $38.5 \pm 19.6$ & $52.7 \pm 24.1$ & $44.3 \pm 15.3$ \\
& CT $(s)$ & $27.3 \pm 15.6$ & $25.9 \pm 12.4$ & $33.0 \pm 16.1$ & $31.3 \pm 10.9$ \\
& CF $(s-1)$ & $1.59 \pm 0.21$ & $1.48 \pm 0.20$ & $1.63 \pm 0.29$ & $1.44 \pm 0.3$ \\
Peanut & CC & $44.6 \pm 23.0$ & $39.5 \pm 20.5$ & $57.0 \pm 24.0$ & $48.7 \pm 12.6$ \\
& CT (s) & $28.1 \pm 13.4$ & $25.5 \pm 12.1$ & $38.9 \pm 16.4$ & $32.5 \pm 9.9$ \\
& CF $(s-1)$ & $1.60 \pm 0.35$ & $1.56 \pm 0.20$ & $1.56 \pm 0.20$ & $1.53 \pm 0.27$ \\
\hline
\end{tabular}

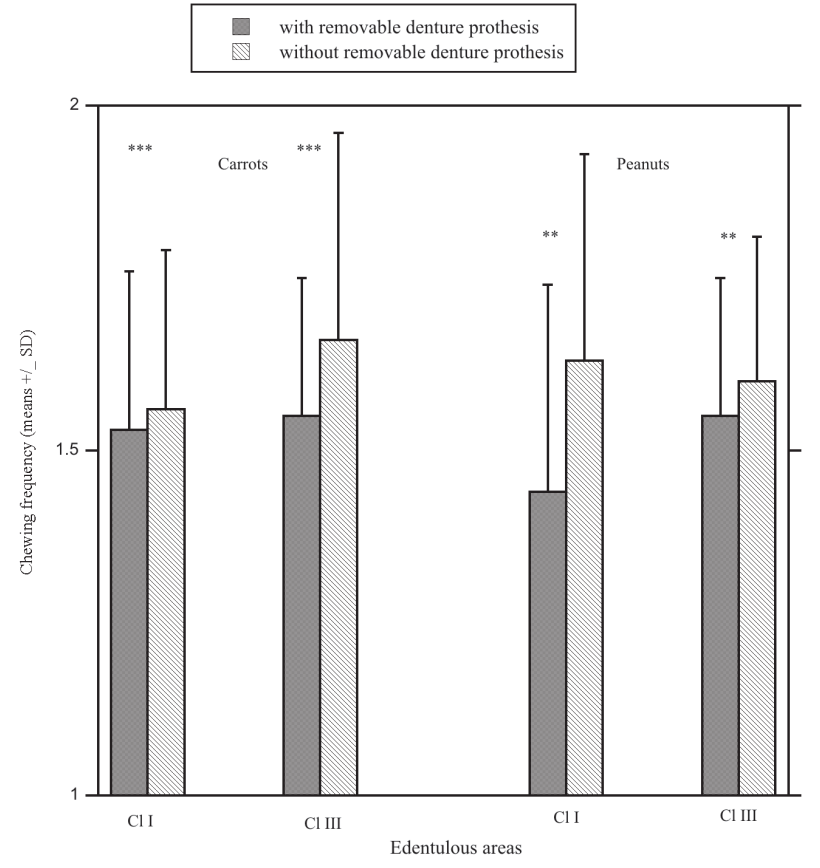

Figure 2- Mean chewing frequency \pm standard deviation of the carrot and peanut with and without removable partial denture prostheses according to the edentulous areas (Kennedy Class). Repeated measure procedure was used for intergroup comparisons $\left({ }^{* *} p<0.001,{ }^{* *} p<0.01\right)$

\section{Kinematic parameters of mastication}

The mean CC, CT, and CF values measured for carrot and peanuts with and without RPDP are presented in Table 1. When comparing mean values of $\mathrm{CC}, \mathrm{CT}$, and $\mathrm{CF}$, controlling for subject with or without RPDP, significant decreases in CC, CT and $\mathrm{CF}$ mean values were shown (RMP procedure, $\mathrm{F}=18$ for $\mathrm{CC}, \mathrm{F}=9$ for $\mathrm{CT}$, and $\mathrm{F}=20$ for $\mathrm{CF} ; \mathrm{p}<0.001$ ). These variations were independent of the type of Kennedy Class and type of food with one exception: the decrease in the chewing frequency with RPDP was dependent of the type of food $(F=7, p<0.05)$ (Figure 2).

\section{DISCUSSION}

This study aimed at evaluating the impact of rehabilitation with removable partial denture prosthesis on chewing parameters. The mastication variables used in this study were chewing frequency and the granulometry measured during the mastication of two test foods: carrots and peanuts. A decrease in the median particle size of the test foods boluses and an increase in the chewing frequency were shown. These results were not dependent of the type of Kennedy Class.

Some limits of this study could be outlined. In particular, the participants had their prostheses in place for at least 2 months and reported no difficulty for eating. However, the experiment was not strictly standardized for the time of prosthesis use. It is possible that a person using oral prosthesis for a longer period could be more adapted, performing oral function better than a person with a recent prosthesis placement. This aspect should be explored in further clinical studies. Furthermore, chewing adaptation to food hardness was not assessed in this experiment. According to previous studies, three viscoelastic model foods, differing in hardness, could be used to explore if RPDP improves adaptability of mastication 7,9 .

Granulometry measurement consists in comparing median particle size or D50. In this study, the D50 value remained above the MNI value, regardless of the type of edentulous areas, or the presence or not of prosthesis. As showed in preceding studies, this value improved with prostheses rehabilitation but insufficiently ${ }^{11,16}$. Thus, it was confirmed, as previously demonstrated in other populations, that the number of functional units control chewing efficiency ${ }^{2,14}$. Effectively, feedback controls are necessary for the formation of the food bolus, allowing modulation of muscular activity. The involvement of these feedback controls varies as a function of the loss of periodontal sensitivity due to edentulous areas. Nonetheless, a completely edentulous patient will retain their 
sensing ability through mucosal, muscular and temporomandibular joint sensitivity. A previous study showed that wearers of removable dentures were losing precision in the control force needed to crush food, a loss directly correlated to the need for a prosthesis ${ }^{9}$. In an intermediate situation, partial edentulous subjects with an impaired dental status could not produce a food bolus with the same particle size distribution as the fully dented patients ${ }^{7}$. The reduction in proprioception due to the lack of dental functional units could not be fully compensated by mucosal sensitivity re-attained when wearing prostheses. However, previous studies showed that implant-related proprioception helped maintaining chewing efficiency ${ }^{3,16}$.

A second variable contributed to the hypothesis that complete chewing function rehabilitation cannot be obtained when using RPDP. The chewing frequency of the food samples in both groups of edentulous subjects (posterior or bounded by remaining teeth) decreased significantly $(p<0.001)$ when subjects wore removable partial denture prosthesis. Chewing frequency is the mastication rhythm linked to a particular food. Some authors specified that chewing frequency is the most reproducible masticatory parameter between tests on the same individual ${ }^{15}$. Consequently, chewing frequency is often used as one of two parameters used to detect chewing deficiency. A lower chewing frequency is therefore a sign of a disturbed mastication function. The results of this study are in agreement with these facts, highlighting a decrease in chewing frequency when missing teeth are replaced by removable partial denture prosthesis. The prosthesis constitutes an artificial element that is more or less stable depending on the type of edentulous areas and the number of missing teeth, as this modifies jaw kinematics.

\section{CONCLUSION}

This study showed that RPDP rehabilitation does improve the ability to reduce the bolus particle size, but is not able to fully restore the masticatory function. The median bolus granulometry values measured without or with prostheses remained above the normative values but the chewing frequency continues to be impaired.

\section{REFERENCES}

\footnotetext{
1- Aras K, Hasanreisoğlu U, Shinogaya T. Masticatory performance, maximum occlusal force, and occlusal contact area in patients with bilaterally missing molars and distal extension removable partial dentures. Int J Prosthodont. 2009;22(2):204-9.

2- Decerle N, Nicolas E, Hennequin M. Chewing deficiencies in adults with multiple untreated carious lesions. Caries Res. 2013;47(4):330-7.
}

3- Grigoriadis A, Johansson RS, Trulsson M. Adaptability of mastication in people with implant-supported bridges. J Clin Periodontol. 2011;38(4):395-404.

4- Hatch JP, Shinkai RS, Sakai S, Rugh JD, Paunovich ED. Determinants of masticatory performance in dentate adults. Arch Oral Biol. 2001;46(7):641-8.

5- Hennequin M, Allison PJ, Veyrune JL, Faye M, Peyron M. Clinical evaluation of mastication: validation of video versus electromyography. Clin Nutr. 2005;24(2):314-20.

6- Kanno T, Carlsson GE. A review of the shortened dental arch concept focusing on the work by the Käyser/Nijmegen group. J Oral Rehabil. 2006;33(11):850-62.

7- Lassauzay C, Peyron MA, Albuisson E, Dransfield E, Woda A. Variability of the masticatory process during chewing of elastic model foods. Eur J Oral Sci. 2000;108(6):484-92.

8- Levin B. Editorial: The 28-tooth syndrome - or should all teeth be replaced? Dent Surv. 1974;50(7):47.

9- Mishellany-Dutour A, Renaud J, Peyron MA, Rimek F, Woda A. Is the goal of mastication reached in young dentates, aged dentates and aged denture wearers? Br J Nutr. 2008;99(1):121-8.

10- N'gom PI, Woda A. Influence of impaired mastication on nutrition. J Prosthet Dent. 2002;87(6):667-73.

11- Nicolas E, Veyrune J, Lassauzay C. A six-month assessment of oral health-related quality of life of complete denture wearers using denture adhesive: a pilot study. J Prosthodont. 2010;19(6):443-8. 12- Nicolas E, Veyrune JL, Lassauzay C, Peyron MA, Hennequin M. Validation of video versus electromyography for chewing evaluation of the elderly wearing a complete denture. J Oral Rehabil. 2007;34(8):566-71.

13- Peyron MA, Lassauzay C, Woda A. Effects of increased hardness on jaw movement and muscle activity during chewing of viscoelastic model foods. Exp Brain Res. 2002;142(1):41-51.

14- Veyrune JL, Miller CC, Czernichow S, Ciangura CA, Nicolas E, Hennequin M. Impact of morbid obesity on chewing ability. Obes Surg. 2008;18(11):1467-72.

15- Veyrune JL, Mioche L. Complete denture wearers: electromyography of mastication and texture perception whilst eating meat. Eur J Oral Sci. 2000;108(2):83-92.

16- Veyrune JL, Opé S, Nicolas E, Woda A, Hennequin M. Changes in mastication after an immediate loading implantation with complete fixed rehabilitation. Clin Oral Investig. 2013;17(4):112734.

17- Witter DJ, van Elteren $P$, Käyser AF, van Rossum MJ. The effect of removable partial dentures on the oral function in shortened dental arches. J Oral Rehabil. 1989;16(1):27-33.

18- Woda A, Foster K, Mishellany A, Peyron MA. Adaptation of healthy mastication to factors pertaining to the individual or to the food. Physiol Behav. 2006;89(1):28-35.

19- Woda A, Nicolas E, Mishellany-Dutour A, Hennequin M, Mazille $M-N$, Veyrune J-L, et al. The masticatory normative indicator. J Dent Res. 2010;89(3):281-5. 